10 Aug 2018

LBL-33946, UCB-PTH-93/12

\title{
Quarkonium Decays and Light Quark Masses
}

\author{
Markus A. Luty* and Raman Sundrum*广 \\ * Theoretical Physics Group \\ Lawrence Berkeley Laboratory \\ 1 Cyclotron Road \\ Berkeley, California 94720 \\ $\dagger$ Department of Physics \\ University of California \\ Berkeley, California 94720
}

\begin{abstract}
The $S U(3)$-violating decays $\Phi^{2 S} \rightarrow \Phi^{1 S} X$, where $X=\pi^{0}$ or $\eta$ and $\Phi=J / \psi$ or $\Upsilon$ have been recently proposed as a means of probing the light quark masses beyond leading order in chiral perturbation theory. We argue that this analysis is incorrect, even in the heavy quark limit. We show that these decays are governed by an infinite number of matrix elements which are not suppressed by any small parameter, and which cannot be computed with our present understanding of QCD. Furthermore, for sufficiently heavy quarks, we show that the decay amplitudes can be organized into a twist expansion, and that the contributions considered in the above proposal are subleading in this expansion. We also explain how these decays nonetheless give a constraint on the light quark masses valid at leading order in the chiral expansion. The decays $\Phi^{1 S} \rightarrow \eta \gamma$ and $\Phi^{2 S} \rightarrow \Phi^{1 S} \pi \pi$ also have contributions from infinitely many operators, contrary to claims in the literature.
\end{abstract}

This work was supported in part by the Director, Office of Energy Research, Office of High Energy and Nuclear Physics, Division of High Energy Physics of the U.S. Department of Energy under contract DE-AC03-76SF00098 and in part by the National Science Foundation under grant PHY90-21139. 


\section{Introduction}

Recently, there has been a renewed interest in the flavor $S U(3)$-violating decays

$$
\Phi^{2 S} \rightarrow \Phi^{1 S} \pi^{0} \quad \text { and } \quad \Phi^{2 S} \rightarrow \Phi^{1 S} \eta
$$

where $\Phi=J / \psi$ or $\Upsilon$, as a probe for the light quark masses beyond leading order in chiral perturbation theory [1][2]. This work is based on ref. [3], where it is argued that the QCD multipole expansion [4][5] can be used to show that

$$
\mathcal{A}\left(\Phi^{2 S} \rightarrow \Phi^{1 S} X\right) \propto\left\langle X\left|\operatorname{tr} G^{\mu \nu} \widetilde{G}_{\mu \nu}\right| 0\right\rangle
$$

where $X=\pi^{0}$ or $\eta$. The basic idea in ref. [3] is that the $\Phi$ is spatially small compared to the wavelength of gluons which are important in the decay process, and that the decay can be computed in terms of the matrix elements of local gluonic operators. This result is supposed to be valid for sufficiently heavy quark masses. The hadronic matrix element in eq. (2) can then be computed using chiral perturbation theory [6][2].

In this paper, we show that eq. (2) is not the leading contribution to the decays in eq. (1): there are an infinite number of operators which contribute whose matrix elements are not suppressed by any small parameter. This result is a simple consequence of the non-locality of the transition amplitude in time. Furthermore, in the heavy quark limit, the decay amplitude can be organized into a twist expansion, and the contribution leading to eq. (2) can be shown to be subleading in this expansion. This invalidates the claim of ref. [2] that the decays in eq. (1) can be used to constrain the light quark masses at next-to-leading order in the chiral expansion. Despite this negative result, we show that the decays (1) can be used to derive a constraint on the light quark masses valid at leading order in the chiral expansion, but not at higher orders. This distinction is important, since the corrections to lowest-order results are substantial, and may even allow $m_{u}=0$, giving an economical solution of the strong $C P$ problem [7].

A similar analysis can be used to show that the decays

$$
\Phi^{2 S} \rightarrow \Phi^{1 S} \pi \pi \quad \text { and } \quad \Phi^{1 S} \rightarrow \eta \gamma
$$

also get contributions from infinitely many operators, contrary to the claims of refs. [3] and [6], respectively.

\section{Quantum Mechanics Analysis}

In this section, we adopt the assumptions and methodology used in ref. [3] to describe the decay in eq. (1). We show that these assumptions lead to the conclusion that these 
decays are governed by an infinite number of matrix elements which are unsuppressed by any small parameter. In the next section, we will argue that this conclusion is much more general than the argument of this section, and that the contributions considered in this section are in fact not the dominant ones.

We will treat the $\Phi$ as a non-relativistic quantum-mechanical bound state. We begin by making the standard assumption that the decay in eq. (1) can be viewed as a twostep process: First the $\Phi^{2 S}$ decays via the emission of two gluons, and then the gluons "hadronize" to form the light pseudoscalar in the final state. The wavelength of the gluons involved is large compared to the spatial size of the $\Phi$, and so gluon emission can be described in terms of a local perturbation hamiltonian involving the gluon fields.

In ref. [3], it is assumed that the leading contribution to the decays in eq. (1) comes from interference between the electric dipole interaction

$$
\delta H_{1}=-g \vec{r} \cdot \vec{E}
$$

and the the spin-dependent interaction

$$
\delta H_{2}=-\frac{g}{2 m_{Q}}(\vec{r} \cdot \vec{D})(\vec{S} \cdot \vec{B})
$$

which arises at higher order in the nonrelativistic expansion. Here, $\vec{r}$ is the separation between the quark and the antiquark, $\vec{S}$ is the total spin operator, and

$$
\vec{E} \equiv-\vec{\nabla} A_{a}^{0} T_{a}, \quad \vec{B} \equiv \vec{\nabla} \times \vec{A}_{a} T_{a}
$$

where

$$
T_{a} \equiv \frac{1}{2}\left(t_{a} \otimes 1+1 \otimes t_{a}^{*}\right)
$$

The matrix element is then given in second-order perturbation theory by

$$
\mathcal{A} \propto\left\langle f\left|\delta H_{1} \frac{1}{\epsilon_{i}-\left(H_{Q \bar{Q}}+H_{g}\right)+i 0+} \delta H_{2}\right| i\right\rangle+(1 \leftrightarrow 2),
$$

where $\epsilon_{i}$ is the energy of the state $|i\rangle, H_{Q \bar{Q}}$ is the hamiltonian of the $Q \bar{Q}$ pair in the QCD-generated potential and $H_{g}$ is the hamiltonian for free gluons. Inserting a complete set of energy eigenstates, we obtain

$$
\mathcal{A} \propto \sum_{\alpha}\left\langle f\left|\delta H_{1} \frac{1}{\epsilon_{i}-H_{Q \bar{Q}}-\omega_{\alpha}+i 0+}\right| \alpha\right\rangle\left\langle\alpha\left|\delta H_{2}\right| i\right\rangle+(1 \leftrightarrow 2),
$$


where $\omega_{\alpha}$ is the energy carried away by the emission of the first gluon. We can expand this to obtain

$$
\begin{aligned}
\mathcal{A} & \propto \sum_{n} \sum_{\alpha}\left\langle f\left|\delta H_{1} \frac{1}{\left(\epsilon_{i}-H_{Q \bar{Q}}+i 0+\right)^{n+1}}\right| \alpha\right\rangle\left\langle\alpha\left|\omega_{\alpha}^{n} \delta H_{2}\right| i\right\rangle+(1 \leftrightarrow 2) \\
& =\sum_{n}\left\langle f\left|\delta H_{1} \frac{1}{\left(\epsilon_{i}-H_{Q \bar{Q}}+i 0+\right)^{n+1}}\left(i \partial_{0}\right)^{n} \delta H_{2}\right| i\right\rangle+(1 \leftrightarrow 2),
\end{aligned}
$$

where the time derivative $\partial_{0}$ is understood to act on the gluon fields in $\delta H_{2}$. We therefore obtain

$$
\begin{aligned}
\mathcal{A}\left(\Phi^{2 S} \rightarrow \Phi^{1 S} X\right) \propto \frac{g^{2}}{2 m_{Q}} \sum_{n}\left\langle\Phi^{1 S}\left|r_{j} \frac{1}{\left(\epsilon_{i}-H_{Q \bar{Q}}+i 0+\right)^{n+1}} r_{k} S_{\ell}\right| \Phi^{2 S}\right\rangle \\
\times\left\langle X\left|E_{j}\left(i D_{0}\right)^{n} D_{k} B_{\ell}+D_{j} B_{\ell}\left(i D_{0}\right)^{n} E_{k}\right| 0\right\rangle
\end{aligned}
$$

where we have included additional terms mandated by gauge invariance by substituting the gauge-covariant derivative $D_{0}$ for the time derivative $\partial_{0}$. Similar results are derived using field-theoretic arguments in ref. [5].

If we expand this result in powers of $H_{Q \bar{Q}}$, eq. (11) has the form of an operator product expansion, with the suppression scale of the higher-dimension operators given by the spacing between excited energy levels $\Delta$. If we keep only the first term in this expansion, we recover the result eq. (2) of ref. [3]. However, the kinematic energy scale for these decays is $M_{2 S}-M_{1 S}$, which is of order $\Delta$, so there is no small parameter suppressing the higher order terms in the series in eq. (11), even in the heavy-quark limit. This is the central point of this paper.

To show explicitly that there is no suppression of higher-order terms in eq. (11), we follow ref. [3] and assume that the spatial and spin parts of the $\Phi$ wavefunction factorize. We can then write eq. (11) as

$$
\begin{aligned}
\mathcal{A}\left(\Phi^{2 S} \rightarrow \Phi^{1 S} X\right) \propto \frac{g^{2}}{2 m_{Q}} \sum_{n} \frac{c_{n}}{\Delta^{n+3}}\left(\vec{\Phi}^{1 S} \times \vec{\Phi}^{2 S}\right)_{j} & \\
& \times\left\langle X\left|E_{k}\left(i D_{0}\right)^{n} D_{k} B_{j}+D_{k} B_{j}\left(i D_{0}\right)^{n} E_{k}\right| 0\right\rangle,
\end{aligned}
$$

where $c_{n}$ is a quantum-mechanical matrix element, and $\vec{\Phi}^{1 S}$ and $\vec{\Phi}^{2 S}$ are polarization vectors for the initial and final quarkonium states. In order to estimate the size of the hadronic matrix element, we write the operator in relativistic notation using the fourvelocity $v$ of the decaying particle:

$$
\mathcal{A}\left(\Phi^{2 S} \rightarrow \Phi^{1 S} X\right) \propto \frac{g^{2}}{2 m_{Q}} \sum_{n} \frac{c_{n}}{\Delta^{n+3}} v_{\mu} \epsilon^{\mu \nu \lambda \rho} \Phi_{\nu}^{1 S} \Phi_{\lambda}^{2 S} v^{\mu} v^{\nu} v^{\lambda_{1}} \cdots v^{\lambda_{n}}\left\langle X\left|\mathcal{O}_{\rho \mu \nu \lambda_{1} \cdots \lambda_{n}}^{(n)}\right| 0\right\rangle
$$


where

$$
\mathcal{O}_{\rho \mu \nu \lambda_{1} \cdots \lambda_{n}}^{(n)} \equiv \operatorname{tr} G_{\sigma(\mu} D_{\lambda_{1}} \cdots D_{\lambda_{n}} D^{\sigma} \widetilde{G}_{\nu) \rho}+\operatorname{tr} D^{\sigma} \widetilde{G}_{\rho(\mu} D_{\lambda_{1}} \cdots D_{\lambda_{n}} G_{\nu) \sigma} .
$$

Here, we use the notation $(\cdots)$ to denote the symmetrization of indices. The hadronic matrix elements can be written

$$
\begin{aligned}
\left\langle X\left|\mathcal{O}_{\rho \mu \nu \lambda_{1} \cdots \lambda_{n}}^{(n)}\right| 0\right\rangle=\Lambda^{2} & {\left[g_{(\mu \nu} p_{\lambda_{1}} \cdots p_{\left.\lambda_{n}\right)} p_{\rho}-(\nu \leftrightarrow \rho)\right] F_{1}^{(n)} } \\
+ & \Lambda^{4}\left[g_{(\mu \nu} g_{\lambda_{1} \lambda_{2}} p_{\lambda_{3}} \cdots p_{\left.\lambda_{n}\right)} p_{\rho}-(\nu \leftrightarrow \rho)\right] F_{2}^{(n)}+\cdots
\end{aligned}
$$

where $p$ is the momentum of $X$. We have explicitly factored out powers of the hadronic scale $\Lambda$ so that all the form factors $F_{j}$ have the same dimension. Since the form factors depend only on $p^{2}=m_{\pi}^{2}$, they are constants determined by low-energy QCD dynamics, and are all expected to be of the same order of magnitude. (Note that since the decay violates $S U(3)$, the form factors $F_{j}$ must be proportional to quark masses.)

Substituting this into eq. (13), we obtain

$$
\mathcal{A}\left(\Phi^{2 S} \rightarrow \Phi^{1 S} X\right) \propto \frac{g^{2}}{2 m_{Q}} \Lambda^{2} \sum_{n} \frac{c_{n}}{\Delta^{n+3}} v_{\mu} \epsilon^{\mu \nu \lambda \rho} \Phi_{\nu}^{1 S} \Phi_{\lambda}^{2 S} p_{\rho}(p \cdot v)^{n} F_{1}^{(n)}+\cdots
$$

Since $p \cdot v \sim \Delta$, we see that the contributions from the higher order terms in this expansion are unsuppressed, even in the heavy quark limit.

Similar arguments which led to eq. (2) were used in ref. [3] to argue that

$$
\mathcal{A}\left(\Phi^{2 S} \rightarrow \Phi^{1 S} \pi \pi\right) \propto\left\langle\pi \pi\left|\operatorname{tr} G^{\mu \nu} G_{\mu \nu}\right| 0\right\rangle
$$

and in ref. [6] to argue that

$$
\mathcal{A}\left(\Phi^{1 S} \rightarrow \eta \gamma\right) \propto\left\langle\eta\left|\operatorname{tr} G^{\mu \nu} \widetilde{G}_{\mu \nu}\right| 0\right\rangle
$$

The considerations above can be used to show that these decays also have unsuppressed contributions from an infinite number of operators.

\section{Effective Field Theory Analysis}

The conclusions of the previous section are not at all unexpected if we consider these decays in an effective field theory language. If we consider interactions of the $\Phi^{1 S}$ state with gluons with energies $E \ll \Delta$, then we can write an effective field theory in which the $\Phi^{1 S}$ is treated as a heavy particle. Higher-dimension terms in this effective lagrangian are suppressed by powers of $\Delta$, the energy required to excite the next excited state. In order to 
consider decays such as those in eq. (1), we must write an effective theory containing fields for both $\Phi^{1 S}$ and $\Phi^{2 S}$ states interacting with gluons. This is not a very useful effective theory, since the higher-dimension terms in this lagrangian are again suppressed by powers of $\Delta$, which is also the kinematic scale for the decays we are interested in. Therefore, we expect that an infinite number of gluonic operators will contribute at the same level to the decay. This situation arises because we are trying to describe a process which is nonlocal (in time) on a scale $\sim 1 / \Delta$ by local operators.

In an effective field theory for $\Phi$ radial transitions, we could immediately write down contributions to the decay such as eq. (13) and reach the conclusions of the previous section. But we can also write terms such as

$$
\mathcal{L}_{\text {eff }}=\frac{c}{\Delta^{4}} v_{\mu} \epsilon^{\mu \nu \lambda \rho} \Phi_{\nu}^{1 S} \Phi_{\lambda}^{2 S} \operatorname{tr}\left(G_{(\alpha}^{\sigma} D_{\lambda_{1}} \cdots D_{\lambda_{n}} D_{\rho} \widetilde{G}_{\beta) \sigma}\right) v^{\alpha} v^{\beta} v^{\lambda_{1}} \cdots v^{\lambda_{n}}+\cdots
$$

There is no symmetry which forbids these terms, so we expect them to be generated at some level. In fact, at higher orders in the non-relativistic expansion, factorization of the spin and spatial parts of the $\Phi$ wavefunction assumed in eq. (12) breaks down, generating the contributions in eq. (19). These terms give rise to contributions to the decay amplitude proportional to

$$
\left\langle X\left|\operatorname{tr}\left(G_{(\alpha}^{\sigma} D_{\lambda_{1}} \cdots D_{\lambda_{n}} D_{\rho} \widetilde{G}_{\beta) \sigma}\right)\right| 0\right\rangle=p_{\alpha} p_{\beta} p_{\lambda_{1}} \cdots p_{\lambda_{n}} p_{\rho} G_{1}+\cdots,
$$

where the form factor has been normalized to have the same dimension as those in eq. (15). In the heavy-quark limit, the contribution of this term to the decay amplitude is enhanced relative to eq. (16) by a factor of

$$
g^{m}\left(\frac{p \cdot v}{\Lambda}\right)^{2} \sim\left(\frac{m_{Q}}{\Lambda}\right)^{2},
$$

up to logarithms of $m_{Q}$, where $\Lambda \sim 1 \mathrm{GeV}$ is a hadronic scale.

In general, for terms in the effective theory of the form

$$
\mathcal{L}_{\text {eff }}=\sum_{n} \frac{c_{n}}{\Delta^{n}} v_{\mu} \epsilon^{\mu \nu \lambda \rho} \Phi_{\nu}^{1 S} \Phi_{\lambda}^{2 S} \mathcal{O}_{\rho}^{(n)}
$$

where $\mathcal{O}^{(n)}$ is a QCD operator, it is easy to see that the leading contributions will come from operators with lowest twist (= dimension-spin). The operator in eq. (15) has twist 4, while the operator in eq. (20) has twist 2 . Thus, the contributions to the decay amplitude in eq. (12) are not among the leading ones in the heavy-quark limit.

The relevance of the twist expansion to the real world is unclear, since the $c$ and $b$ quarks are not sufficiently heavy to trust heavy-quark results without reservation, and in any case the hadronic matrix elements of the operators in question are unknown. 


\section{Determination of the Light Quark Masses}

Our main motivation for this work is the fact that ref. [2] argued that the decays in eq. (1) can be used to give a constraint on the light quark masses beyond leading order in the chiral expansion. We have argued that these decays are not dominated by the matrix elements in eq. (2), so it may seem mysterious that the constraint obtained in ref. [2] is consistent with other determinations of the light quark mass ratios. However, it is easy to see that the decays (1) give a constraint on the light quark masses valid at leading order in the chiral expansion.

The point is that the decay is forbidden in the limit of exact vector $S U(3)$. However, $S U(3)$ is broken explicitly by the quark masses, so the leading contribution to the amplitude can be written

$$
\mathcal{A}\left(\Phi^{2 S} \rightarrow \Phi^{1 S} X\right) \propto \operatorname{tr}\left(M_{q} T_{X}\right),
$$

where $M_{q}$ is the light quark mass matrix and $T_{X}$ is the $S U(3)$ generator corresponding to the pseudoscalar in the final state. (We can use chiral invariance to show that a similar term does not exist due to electromagnetic breaking of $S U(3)$.) This form of the amplitude immediately gives the leading order constraint on the light quark masses used in ref. [2]. Similar arguments to those given above can be found in ref. [1].

\section{Conclusions}

We have shown that the decays in eq. (1) cannot be used to constrain the light quark masses beyond leading order. These decays are governed by an infinite number of operators which are unsuppressed by any small parameter even in the heavy quark limit. Furthermore, for sufficiently heavy quark masses, the leading contributions to the decay come from operators of twist 2, while the contribution discussed in ref. [3] has twist 4.

The use of the decays in eq. (1) to determine the light quark masses has also been criticized in ref. [8]. However, this paper assumes that the leading contribution to the decay comes from the first term of eq. (12), and criticizes the quantitative accuracy of the heavy quark and $1 / N_{\text {color }}$ expansion used in ref. [3].

\section{Acknowlegements}

We would like to thank M. E. Peskin and M. Suzuki for discussions. This work was supported in part by the Director, Office of Energy Research, Office of High Energy and Nuclear Physics, Division of High Energy Physics of the U.S. Department of Energy under contract DE-AC03-76SF00098 and in part by the National Science Foundation under grant PHY90-21139. 


\section{References}

[1] B. L. Ioffe and M. A. Shifman, Phys. Lett. 95B, 99 (1980).

[2] J. F. Donoghue and D. Wyler, Phys. Rev. D45, 892 (1992); J. F. Donoghue, B. R. Holstein, and D. Wyler, Phys. Rev. Lett. 69, 3444 (1992).

[3] M. Voloshin and V. Zakharov, Phys. Rev. Lett. 45, 688 (1980).

[4] K. Gottfried, Phys. Rev. Lett. 40, 598 (1978).

[5] M. E. Peskin, Nucl. Phys. B156, 365 (1979); M. E. Peskin and G. Bhanot, Nucl. Phys. B156, 391 (1979).

[6] V. A. Novikov, M. A. Shifman, A. I. Vainshtein, and V. I. Zakharov, Nucl. Phys. B165, 55 (1980).

[7] The debate over whether $m_{u}=0$ is allowed is ongoing. See D. B. Kaplan and A. V. Manohar Phys. Rev. Lett. 56, 2004 (1986); H. Leutwyler, Nucl. Phys. B337, 108 (1990);

K. Choi, Nucl. Phys. B383, 58 (1992); see also ref. [2].

[8] K. Choi, Phys. Lett. 292B, 159 (1992). 